Honam Mathematical J. 34 (2012), No. 4, pp. 559-570

http://dx.doi.org/10.5831/HMJ.2012.34.4.559

\title{
NEIGHBORHOOD STRUCTURES IN ORDINARY SMOOTH TOPOLOGICAL SPACES
}

\author{
Jeong Gon Lee, Pyung Ki Lim and Kul HuR*
}

\begin{abstract}
We construct a new definition of a base for ordinary smooth topological spaces and introduce the concept of a neighborhood structure in ordinary smooth topological spaces. Then, we state some of their properties which are generalizations of some results in classical topological spaces.
\end{abstract}

\section{Introduction}

In 1985, Sostak [6] defined a fuzzy topology $\tau$ on a nonempty set $X$ as a mapping $\tau: I^{X} \rightarrow I$ satisfying three axioms, where $I^{X}$ denotes the set of all fuzzy sets in $X$. He considered the degree of openness of fuzzy sets, gave some basic rules and proved how such an extension can be done. In 1992, Chattopadhyay et al. [1] studied the fuzzy topological spaces in Sostak's sense. In the same year, Ramadan [5] introduced similar concepts under the name of smooth topological spaces working in terms of lattices $L$ and $L^{\prime}$ instead of $I=[0,1]$. In particular, Demirci [3] introduced the concepts of neighborhood structures in smooth topological spaces. Moreover, Ying [7] investigated fuzzifying topological spaces (called ordinary smooth topological spaces by Hur et al. [4]) considering of degree of openness of ordinary subsets. Recently, Chae et al. [2] constructed the set $\mathrm{OST}(\mathrm{X})$ of all ordinary smooth topologies on a set $X$ and studied it in the sense of a lattice.

In this paper,we construct a new definition of a base for ordinary smooth topological spaces and introduce the concept of a neighborhood structure in ordinary smooth topological spaces. Then, we state some

Received September 19, 2012. Accepted December 11, 2012.

2010 Mathematics Subject Classification. 54A40.

Key words and phrases. ordinary smooth topological space, ordinary smooth, base, ordinary neighborhood system.

${ }^{*}$ Corresponding Author 
of their properties which are generalizations of some results in classical topological spaces.

\section{Preliminaries}

Let $2=\{0,1\}$ and let $2^{X}$ denote the set of all ordinary subsets of $X$. Definition 2.1[4]. Let $X$ be an nonempty set. Then a mapping $\tau$ : $2^{X} \rightarrow I$ is called an ordinary smooth topology (in short, ost) on $X$ or a gradation of openness of ordinary subsets of $X$ if $\tau$ satisfies the following axioms:

$\left(\mathrm{OST}_{1}\right) \tau(\emptyset)=\tau(X)=1$

$\left(\mathrm{OST}_{2}\right) \tau(A \cap B) \geq \tau(A) \wedge \tau(B), \forall A, B \in 2^{X}$.

$\left(\mathrm{OST}_{3}\right) \tau\left(\bigcup_{\alpha \in \Gamma} A_{\alpha}\right) \geq \bigwedge_{\alpha \in \Gamma} \tau\left(A_{\alpha}\right), \forall\left\{A_{\alpha}\right\} \subset 2^{X}$.

The pair $(X, \tau)$ is called an ordinary smooth topological space (in short, osts). We will denote the set of all osts's on $X$ as $\operatorname{OST}(\mathrm{X})$.

Remark 2.2. Ying [7] called the mapping $\tau: 2^{X} \rightarrow I$ [resp. $\tau: I^{X} \rightarrow 2$ and $\left.\tau: I^{X} \rightarrow I\right]$ satisfying the axioms in Definition 2.1 as a fuzzyfying topology [resp. fuzzy topology and bifuzzy topology] on $X$.

Remark 2.3. If $I=2$, then Definition 2.1 coincides with the known definition of the classical topology.

Definition 2.4. Let $X$ be a nonempty set. Then a mapping $\mathcal{C}: 2^{X} \rightarrow I$ is called an ordinary smooth cotopology (in short, osct) on $X$ or a gradation of closedness of ordinary subsets of $X$ if $\mathcal{C}$ satisfies the following axioms :

$\left(\mathrm{OSCT}_{1}\right) \mathcal{C}(\emptyset)=\mathcal{C}(X)=1$

$\left(\mathrm{OSCT}_{2}\right) \mathcal{C}(A \cup B) \geq \mathcal{C}(A) \wedge \mathcal{C}(B), \forall A, B \in 2^{X}$.

$\left(\mathrm{OSCT}_{3}\right) \mathcal{C}\left(\bigcap_{\alpha \in \Gamma} A_{\alpha}\right) \geq \bigwedge_{\alpha \in \Gamma} \mathcal{C}\left(A_{\alpha}\right), \forall\left\{A_{\alpha}\right\} \subset 2^{X}$.

The pair $(X, \mathcal{C})$ is called an ordinary smooth cotopological space (in short, oscts). We will denote the set of all oscts's on $X$ as $\operatorname{OSCT}(X)$.

Remark 2.5. If $I=2$, then Definition 2.4 also coincides with the known definition of the classical topology.

The following is the immediate result of Definition 2.1 and 2.4. 
Result 2.A [4, Proposition 2.7]. Let $X$ be a nonempty set. We define two mappings $f: \operatorname{OST}(\mathrm{X}) \rightarrow \operatorname{OSCT}(\mathrm{X})$ and $g: \operatorname{OSCT}(\mathrm{X}) \rightarrow \operatorname{OST}(\mathrm{X})$ as follows, respectively :

and

$$
[f(\tau)](A)=\tau\left(A^{c}\right), \forall \tau \in \operatorname{OST}(X), \forall A \in 2^{X}
$$$$
[g(\mathcal{C})](A)=\mathcal{C}\left(A^{c}\right), \forall \mathcal{C} \in \operatorname{OSCT}(X), \forall A \in 2^{X} .
$$

Then $f$ and $g$ are well-defined. Furthermore $g \circ f=i d_{\mathrm{OST}(\mathrm{X})}$ and $f \circ g=i d_{\mathrm{OSCT}}(\mathrm{X})$.

Remark 2.6. Let $f(\tau)=\mathcal{C}_{\tau}$ and $g(\mathcal{C})=\tau_{\mathcal{C}}$. Then, Result 2.A, we can easily see that $\tau_{\mathcal{C}_{\tau}}=\tau$ and $\mathcal{C}_{\tau_{\mathcal{C}}}=\mathcal{C}$.

Definition 2.7[4]. Let $(X, \tau)$ be an osts and let $r \in I$. Then we define two ordinary subsets of $X$ as follows :

and

$$
[\tau]_{r}=\left\{A \in 2^{X}: \tau(A) \geq r\right\}
$$

$[\tau]_{r}^{*}=\left\{A \in 2^{X}: \tau(A)>r\right\}$.

We call these the $r$-level set and the strong $r$-level set of $\tau$, respectively.

It is clear that $[\tau]_{0}=2^{X}$, the classical discrete topology on $X$ and $[\tau]_{1}^{*}=\emptyset$. Also it can be easily seen that $[\tau]_{r}^{*} \subset[\tau]_{r}$ for each $r \in I$.

Result 2.B[4, Proposition 2.10]. Let $(X, \tau)$ be an osts and let $T(X)$ be the set of all classical topologies on $X$. Then :
(a) $[\tau]_{r} \in \mathrm{T}(X), \forall r \in I$.
$(\text { a) })^{\prime}[\tau]_{r}^{*} \in \mathrm{T}(X), \forall r \in I_{1}$.
(b) For any $r, s \in I$, if $r \leq s$, then $[\tau]_{s} \subset[\tau]_{r}$ and $[\tau]_{s}^{*} \subset[\tau]_{r}^{*}$.
(c) $[\tau]_{r}=\bigcap_{s<r}[\tau]_{s}, \forall r \in I_{0}$.
$(\mathrm{c})^{\prime}[\tau]_{r}^{*}=\bigcup_{s>r}[\tau]_{s}^{*}, \forall r \in I_{1}$, where $I_{0}=(0,1]$ and $I_{1}=[0,1)$.

\section{Main Results}

For a mapping $t: 2^{X} \rightarrow I$ and $r \in I_{1}$, let us define the family $[t]_{r}^{*}=\left\{A \in 2^{X}: t(A)>r\right\}$ which will play an important role in our study. From Result 2.B, it is clear that if $t \in \mathrm{OST}(\mathrm{X})$, then $[t]_{r}^{*} \in T(X)$. 
Definition 3.1. Let $(X, \tau)$ be an ordinary smooth topological space. Then a mapping $\beta: 2^{X} \rightarrow I$ is called an ordinary smooth base for $\tau$ if $[\beta]_{r}^{*}$ is a classical base for $[\tau]_{r}^{*}$.

Let $(X, T)$ be a classical topological space and for each $p \in X$ and let $\mathcal{N}_{T}(p)$ denote the classical neighborhood system of $p$.

The following is the characterization of Definition 3.1.

Theorem 3.2. Let $(X, \tau)$ be an ordinary smooth topological space. Then a mapping $\beta: 2^{X} \rightarrow I$ is an ordinary smooth base for $\tau$ if and only if for each $r \in I_{1}$ and each $p \in X$, if $A \in \mathcal{N}_{[\tau]_{r}^{*}}(p)$, then there exists $B \in[\beta]_{r}^{*}$ such that $p \in B \subset A$.

Proof. $(\Rightarrow)$ Suppose $\beta: 2^{X} \rightarrow I$ is an ordinary smooth base for $\tau$. Then, by Definition 3.1, $[\beta]_{r}^{*}$ is a classical base for $[\tau]_{r}^{*}$ for each $r \in I_{1}$. For each $p \in X$, let $A \in \mathcal{N}_{[\tau]_{r}^{*}}(p)$. Then there exists $U \in[\tau]_{r}^{*}$ such that $p \in U \subset A$. Since $U \in[\tau]_{r}^{*}$, there exists $\beta_{0} \subset[\beta]_{r}^{*}$ such that $U=\cup \beta_{0}$. Since $p \in U, p \in \cup \beta_{0}$. Thus there exists $B \in \beta_{0}$ such that $p \in B \subset U$. So there exists $B \in[\beta]_{r}^{*}$ such that $p \in B \subset A$. $(\Leftarrow)$ Suppose the necessary condition holds. Assume that the mapping $\beta: 2^{X} \rightarrow I$ is not an ordinary smooth base for $\tau$. Then, by Definition 3.1, there exist $r_{0} \in I_{1}$ and $A \in[\tau]_{r_{0}}^{*}$ such that $A \neq \cup \beta^{\prime}, \forall \beta^{\prime} \subset[\beta]_{r_{0}}^{*}$. Consider the family $\beta^{*}=\left\{B \in[\beta]_{r_{0}}^{*}: B \subset A\right\}$ and let $G=\cup \beta^{*}$. Then clearly $A \neq G$. Thus there exists $p \in X$ such that $p \in A$ and $p \notin G$. Since $A \in[\tau]_{r_{0}}^{*}$ and $p \in A, A \in \mathcal{N}_{[\tau]_{r_{0}}^{*}}(p)$. By hypothesis, there exists $B \in[\beta]_{r_{0}}^{*}$ such that $p \in B \subset A$. By the definition of $\beta^{*}, B \in \beta^{*}$. So $B \subset G$. Since $p \notin G$, $p \notin B$. This is a contradiction. Hence the mapping $\beta: 2^{X} \rightarrow I$ is an ordinary smooth base for $\tau$. This completes the proof.

The following is another characterization of Definition 3.1.

Theorem 3.3. Let $(X, \tau)$ be an ordinary smooth topological space. Then a mapping $\beta: 2^{X} \rightarrow I$ is an ordinary smooth base for $\tau$ if and only if $\tau(A) \leq \bigvee\left\{\beta(B): B \in 2^{X}\right.$ and $\left.\mathrm{p} \in \mathrm{B} \subset \mathrm{A}\right\}$ for each $A \in 2^{X}$ with $p \in A$.

Proof. $(\Rightarrow)$ : Suppose $\beta: 2^{X} \rightarrow I$ is an ordinary smooth base for $\tau$. Let $A \in 2^{X}$ with $p \in A$. Then clearly $\tau(A)=0$ or $\tau(A) \neq 0$.

Case (i) : Suppose $\tau(A)=0$. Then the required inequality is obvious.

Case (ii) : Suppose $\tau(A)=r>0$. Let $\epsilon>0$ be arbitrary such that $\epsilon \leq r$. Then clearly $\tau(A)>r-\epsilon$, i.e., $A \in[\tau]_{r-\epsilon}^{*}$. Thus $A \in \mathcal{N}_{[\tau]_{r-\epsilon}^{*}}(p)$.

By Theorem 3.2, there exists $B \in[\beta]_{r-\epsilon}^{*}$ such that $p \in B \subset A$. So

$$
\bigvee\left\{\beta(B): B \in 2^{X} \text { and } \mathrm{p} \in \mathrm{B} \subset \mathrm{A}\right\}>\mathrm{r}-\epsilon
$$


Since $\epsilon>0$ is arbitrary,

$$
\bigvee\left\{\beta(B): B \in 2^{X} \quad \text { and } \quad \mathrm{p} \in \mathrm{B} \subset \mathrm{A}\right\} \geq \mathrm{r}=\tau(\mathrm{A}) .
$$

Hence, in any cases, the required inequality holds.

$(\Leftarrow)$ : Suppose the necessary condition holds. Let $r \in I_{1}$ and let $U \in[\tau]_{r}^{*}$ such that $p \in U$ for each $p \in X$. Then, by the hypothesis,

$$
\alpha<\tau(U) \leq \bigvee\left\{\beta(B): B \in 2^{X} \text { and } \mathrm{p} \in \mathrm{B} \subset \mathrm{U}\right\} .
$$

Thus there exists $B \in 2^{X}$ such that $p \in B \subset U$ and $\beta(B)>\alpha$. So $B \in[\beta]_{\alpha}^{*}$ and $p \in B \subset U$, i.e., the necessary condition of Theorem 3.2 is satisfied. Hence, by Theorem 3.2, $\beta$ is an ordinary smooth base for $\tau$. This completes the proof.

Definition 3.4. Let $(X, \tau)$ be an ordinary smooth topological space and let $p \in X$. Then a mapping $N_{p}: 2^{X} \rightarrow I$ is called the ordinary smooth neighborhood system of $p$ w.r.t. $\tau$ if $\left[N_{p}\right]_{r}^{*}=\mathcal{N}_{[\tau]_{r}^{*}}(p)$, for each $r \in I_{1}$. In this case, we will call $N_{p}(A)$ as the degree of neighborhood of $A$ to $p$ and each member of $\left[N_{p}\right]_{r}^{*}$ is called an ordinary smooth neighborhood of $p$.

The following is the characterization of Definition 3.4.

Theorem 3.5. Let $(X, \tau)$ be an ordinary smooth topological space and let $p \in X$ be fixed. Then a mapping $N_{p}: 2^{X} \rightarrow I$ is the ordinary smooth neighborhood system of $p$ if and only if for each $A \in 2^{X}$,

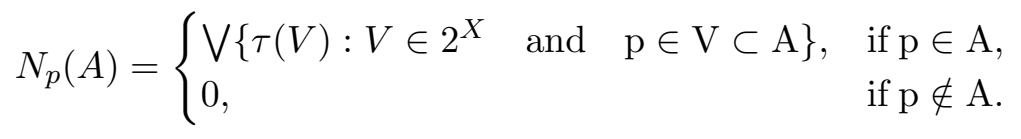

Proof. $(\Rightarrow)$ : Suppose $N_{p}: 2^{X} \rightarrow I$ is the ordinary smooth neighborhood system of $p$ w.r.t. $\tau$ and let $A \in 2^{X}$. Then clearly $p \in A$ or $p \notin A$.

Case (i) Suppose $p \notin A$ and $N_{p}(A)>0$. Then, from the hypothesis and Definition 3.4, there exists $U \in[\tau]_{0}^{*}$ such that $p \in U \subset A$. Thus $p \in A$. This is a contradiction. So $N_{p}(A)=0$.

Case (ii) Suppose $p \in A$. Then we may have $N_{p}(A)=0$ or $N_{p}(A)>0$. If $N_{p}(A)=0$, then it is obvious that

$$
N_{p}(A)=0 \leq \bigvee\left\{\tau(V): V \in 2^{X} \quad \text { and } \quad \mathrm{p} \in \mathrm{V} \subset \mathrm{A}\right\} .
$$

Furthermore, assume that

$$
\bigvee\left\{\tau(V): V \in 2^{X} \text { and } \mathrm{p} \in \mathrm{V} \subset \mathrm{A}\right\}=\beta>0
$$


Then there exists $V \in 2^{X}$ such that $\tau(V)>0$ and $p \in V \subset A$. Thus, by the hypothesis and Definition 3.4, $A \in\left[N_{p}\right]_{0}^{*}$, i.e., $N_{p}(A)>0$. This is a contradiction. So, for the case $N_{p}(A)=0$, we have

$$
N_{p}(A)=\bigvee\left\{\tau(V): V \in 2^{X} \quad \text { and } \quad \mathrm{p} \in \mathrm{V} \subset \mathrm{A}\right\}=0 .
$$

Then, suppose $N_{p}(A)=r>0$. Let $\epsilon>0$ be arbitrary such that $\epsilon \leq r$. Then $N_{p}(A)>r-\epsilon$, i.e., $A \in\left[N_{p}\right]_{r-\epsilon}^{*}$. Since $N_{p}$ is an ordinary smooth neighborhood system of $p$, there exists $U \in[\tau]_{r-\epsilon}^{*}$ such that $p \in U \subset A$. Thus

$$
\bigvee\left\{\tau(V): V \in 2^{X} \quad \text { and } \quad \mathrm{p} \in \mathrm{V} \subset \mathrm{A}\right\}>\mathrm{r}-\epsilon .
$$

Since $\epsilon>0$ is arbitrary,

$$
\bigvee\left\{\tau(V): V \in 2^{X} \quad \text { and } \quad \mathrm{p} \in \mathrm{V} \subset \mathrm{A}\right\} \geq \mathrm{r}=\mathrm{N}_{\mathrm{p}}(\mathrm{A})
$$

On the other hand, let $\bigvee\left\{\tau(V): V \in 2^{X}\right.$ and $\left.\mathrm{p} \in \mathrm{V} \subset \mathrm{A}\right\}=\mathrm{s}$. Then clearly $s>0$. Let $\epsilon>0$ be arbitrary such that $\epsilon \leq s$. Then there exists $V \in 2^{X}$ such that $\tau(V)>s-\epsilon$ and $p \in V \subset A$. Thus $V \in[\tau]_{s-\epsilon}^{*}$ and $p \in V \subset A$. Thus, by the hypothesis, $A \in\left[N_{p}\right]_{s-\epsilon}^{*}$. So $N_{p}(A)>s-\epsilon$. Since $\epsilon>0$ is arbitrary,

$$
N_{p}(A) \geq s=\bigvee\left\{\tau(V): V \in 2^{X} \quad \text { and } \quad \mathrm{p} \in \mathrm{V} \subset \mathrm{A}\right\} .
$$

Hence, by (3.1) and (3.2).

$$
N_{p}(A)=\bigvee\left\{\tau(V): V \in 2^{X} \quad \text { and } \quad \mathrm{p} \in \mathrm{V} \subset \mathrm{A}\right\} \quad \text { for } \quad \mathrm{N}_{\mathrm{p}}(\mathrm{A})>0
$$

This completes the proof of the necessity.

$(\Leftarrow)$ : Suppose the mapping $N_{p}: 2^{X} \rightarrow I$ is given by

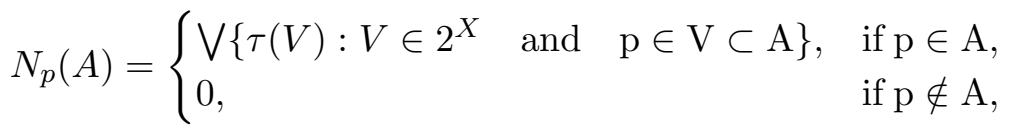

for each $A \in 2^{X}$.

For each $r \in I_{1}$, let $U \in\left[N_{p}\right]_{r}^{*}$, i.e., $N_{p}(U)>r$. Then, from the hypothesis,

$$
r<N_{p}(U)=\bigvee\left\{\tau(V): V \in 2^{X} \quad \text { and } \quad \mathrm{p} \in \mathrm{V} \subset \mathrm{U}\right\}
$$

Thus there exists $V \in 2^{X}$ such that $\tau(V)>r$ and $p \in V \subset U$. So $V \in[\tau]_{r}^{*}$ and $p \in V \subset U$, i.e., $U \in \mathcal{N}_{[\tau]_{r}^{*}}(p)$. Hence $\left[N_{p}\right]_{r}^{*} \subset \mathcal{N}_{[\tau]_{r}^{*}}(p)$.

Now let $r \in I_{1}$ and let $U \in \mathcal{N}_{[\tau]_{r}^{*}}(p)$. Then there exists $B \in[\tau]_{r}^{*}$ such that $p \in B \subset U$. Thus $\tau(B)>r$ and $p \in B \subset U$. So $N_{p}(U)=$ $\bigvee\left\{\tau(V): V \in 2^{X}\right.$ and $\left.\mathrm{p} \in \mathrm{V} \subset \mathrm{U}\right\}>$ r, i.e., $U \in\left[N_{p}\right]_{r}^{*}$. Hence 
$\mathcal{N}_{[\tau]_{r}^{*}}(p) \subset\left[N_{p}\right]_{r}^{*}$. Therefore $\left[N_{p}\right]_{r}^{*}=\mathcal{N}_{[\tau]_{r}^{*}}(p)$ for each $r \in I_{1}$, i.e., $N_{p}$ is the ordinary smooth neighborhood system of $p$ w.r.t. $\tau$.

The following is another characterization of Definition 3.4.

Theorem 3.6. Let $(X, \tau)$ be an ordinary smooth topological space and let $\beta: 2^{X} \rightarrow I$ be an ordinary smooth base for $\tau$. Then a mapping $N_{p}: 2^{X} \rightarrow I$ is the ordinary smooth neighborhood system of $p$ w.r.t. $\tau$ if and only if for each $A \in 2^{X}$,

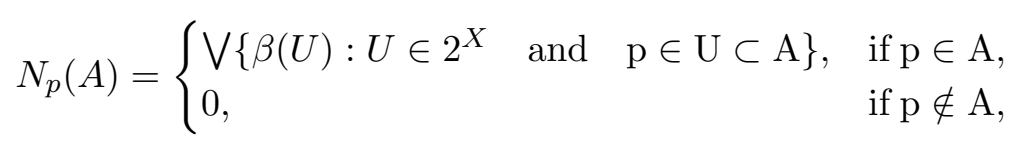

where $p \in X$.

Proof. By considering Theorem 3.2 and Definition 3.4, we can easily obtain that a mapping $N_{p}: 2^{X} \rightarrow I$ is the ordinary smooth neighborhood system of $p$ w.r.t. $\tau$ if and only if $\left[N_{p}\right]_{r}^{*}=\left\{U \in 2^{X}: \exists B \in[\beta]_{r}^{*}\right.$ such that $p \in B \subset U\}$, for each $r \in I_{1}$. Using this equivalence, the proof is completed in a way similar to that of Theorem 3.5.

Proposition 3.7. Let $(X, \tau)$ be an ordinary smooth topological space and let $p \in X$. If the mapping $N_{p}: 2^{X} \rightarrow I$ is the ordinary smooth neighborhood system of $p$ w.r.t. $\tau$, then the followings hold:

$\left(\mathrm{OSN}_{1}\right)$ If $N_{p}(U)>0$, then $p \in U$, where $U \in 2^{X}$.

$\left(\mathrm{OSN}_{2}\right) \bigvee\left\{N_{p}(U): U \in 2^{X}\right\}=1$

$\left(\mathrm{OSN}_{3}\right) N_{p}\left(U_{1} \cap U_{2}\right) \geq N_{p}\left(U_{1}\right) \wedge N_{p}\left(U_{2}\right), \quad \forall U_{1}, U_{2} \in 2^{X}$.

$\left(\mathrm{OSN}_{4}\right)$ If $U_{1} \subset U_{2}$ and $U_{1}, U_{2} \in 2^{X}$, then $N_{p}\left(U_{1}\right) \leq N_{p}\left(U_{2}\right)$.

$\left(\mathrm{OSN}_{5}\right) \forall U \in 2^{X}, N_{p}(U) \leq \bigvee\left\{N_{p}(V) \wedge\left(\bigwedge_{e \in V} N_{e}(V)\right): V \in 2^{X}\right.$ and $\mathrm{V} \subset \mathrm{U}\}$. 3.5 .

Proof. $\left(\mathrm{OSN}_{1}\right),\left(\mathrm{OSN}_{2}\right)$ and $\left(\mathrm{OSN}_{4}\right)$ follows directly from Theorem

$\left(\mathrm{OSN}_{3}\right)$ Let $U_{1}, U_{2} \in 2^{X}$.

Case (i): Suppose $N_{p}\left(U_{1}\right)=0$ or $N_{p}\left(U_{2}\right)=0$. Then the required inequality is obvious.

Case (ii): Suppose $N_{p}\left(U_{1}\right)=r_{1}>0$ and $N_{p}\left(U_{2}\right)=r_{2}>0$. Let $\epsilon>0$ be arbitrary such that $\epsilon \leq r_{1} \wedge r_{2}$. Then

$$
N_{p}\left(U_{1}\right)>r_{1}-\epsilon \geq 0
$$

and

$$
N_{p}\left(U_{2}\right)>r_{2}-\epsilon \geq 0 .
$$


By Definition 3.4, there exist $T_{1}, T_{2} \in 2^{X}$ such that

$$
\tau\left(T_{1}\right)>r_{1}-\epsilon \text { and } \mathrm{p} \in \mathrm{T}_{1} \subset \mathrm{U}_{1}
$$

and

$$
\tau\left(T_{2}\right)>r_{2}-\epsilon \text { and } \mathrm{p} \in \mathrm{T}_{2} \subset \mathrm{U}_{2} .
$$

Thus

$$
\begin{aligned}
\tau\left(T_{1} \cap T_{2}\right) & \geq \tau\left(T_{1}\right) \wedge \tau\left(T_{2}\right) \quad[\text { Since } \tau \in \mathrm{OST}(\mathrm{X})] \\
& >\left(r_{1}-\epsilon\right) \wedge\left(r_{2}-\epsilon\right) \\
& =\left(r_{1} \wedge r_{2}\right)-\epsilon .
\end{aligned}
$$

and

$$
p \in T_{1} \cap T_{2} \subset U_{1} \cap U_{2} .
$$

So, by the hypothesis,

$$
N_{p}\left(U_{1} \cap U_{2}\right)>\left(r_{1} \wedge r_{2}\right)-\epsilon .
$$

Since $\epsilon>0$ is arbitrary,

$$
N_{p}\left(U_{1} \cap U_{2}\right) \geq r_{1} \wedge r_{2}=N_{p}\left(U_{1}\right) \wedge N_{p}\left(U_{2}\right) .
$$

$\left(\mathrm{OSN}_{5}\right)$ Let $U \in 2^{X}$.

Case (i): Suppose $N_{p}(U)=0$. Then the required inequality is obvious.

Case (ii): Suppose $N_{p}(U)=r>0$. Let $\epsilon>0$ be arbitrary such that $\epsilon \leq r$. Then $N_{p}(U)>r-\epsilon$. Thus, by Definition 3.4, there exists $V_{0} \in 2^{X}$ and $\tau\left(V_{0}\right)>r-\epsilon$ and $p \in V_{0} \subset U$. Since $V_{0} \subset V_{0}$ and $\tau\left(V_{0}\right)>r-\epsilon$, $N_{e}\left(V_{0}\right)>r-\epsilon$ for each $e \in V_{0}$. So $\bigwedge_{e \in V_{0}} N_{e}\left(V_{0}\right) \geq r-\epsilon$.

On the other hand, in particular, $N_{p}\left(V_{0}\right)>r-\epsilon$. Thus

$$
\begin{aligned}
& \bigvee\left\{N_{p}(V) \wedge\left(\bigwedge_{e \in V} N_{e}(V)\right): V \in 2^{X} \text { and } \mathrm{V} \subset \mathrm{U}\right\} \\
\geq & N_{p}\left(V_{0}\right) \wedge\left(\bigwedge_{e \in V_{0}} N_{e}\left(V_{0}\right)\right) \\
\geq & r-\epsilon .
\end{aligned}
$$

Since $\epsilon>0$ is arbitrary,

$$
\begin{aligned}
N_{p}(U) & =r \\
& \leq \bigvee\left\{N_{p}(V) \wedge\left(\bigwedge_{e \in V} N_{e}(V)\right): V \in 2^{X} \quad \text { and } \quad \mathrm{V} \subset \mathrm{U}\right\}
\end{aligned}
$$

This completes the proof. 
Proposition 3.8. Let a mapping $N_{p}: 2^{X} \rightarrow I$ satisfy the conditions $\left(\mathrm{OSN}_{1}\right) \sim\left(\mathrm{OSN}_{5}\right)$, where $p \in X$. We define the mapping $\tau: 2^{X} \rightarrow I$ as follows: For each $U \in 2^{X}$,

$$
\tau(U)= \begin{cases}1, & \text { if } \mathrm{U}=\emptyset \\ \bigwedge_{e \in U} N_{e}(U), & \text { otherwise }\end{cases}
$$

Then $\tau \in \operatorname{OST}(\mathrm{X})$. Moreover, the mapping $N_{p}$ is exactly the ordinary smooth neighborhood system of $p$ w.r.t. $\tau$.

Proof. It is obvious that $\tau(\emptyset)=1$. By $\left(\mathrm{OSN}_{2}\right), \bigvee\left\{N_{p}(U): U \in\right.$ $\left.2^{X}\right\}=1$. By $\left(\mathrm{OSN}_{4}\right), N_{p}(U) \leq N_{p}(X)$ for each $U \in 2^{X}$. Thus

$$
\bigvee\left\{N_{p}(U): U \in 2^{X}\right\}=N_{p}(X)=1, \quad \forall p \in X .
$$

So $\tau(U)=\bigwedge_{p \in X} N_{p}(X)=1$. Hence $\tau$ satisfies the condition $\left(\mathrm{OST}_{1}\right)$.

Let $U_{1}, U_{2} \in 2^{X}$. If $U_{1} \cap U_{2}=\emptyset$, then it is obvious that $\tau\left(U_{1} \cap U_{2}\right)=$ $1 \geq \tau\left(U_{1}\right) \wedge \tau\left(U_{2}\right)$. Now suppose $U_{1} \cap U_{2} \neq \emptyset$. Then

$$
\begin{aligned}
\tau\left(U_{1} \cap U_{2}\right) & =\bigwedge_{e \in U_{1} \cap U_{2}} N_{e}\left(U_{1} \cap U_{2}\right) \\
& \geq \bigwedge_{e \in U_{1} \cap U_{2}}\left[N_{e}\left(U_{1}\right) \wedge N_{e}\left(U_{2}\right)\right] \quad\left[\mathrm{By}\left(\mathrm{OSN}_{1}\right)\right] \\
& =\left(\bigwedge_{e \in U_{1} \cap U_{2}} N_{e}\left(U_{1}\right)\right) \wedge\left(\bigwedge_{e \in U_{1} \cap U_{2}} N_{e}\left(U_{2}\right)\right) \\
& \geq\left(\bigwedge_{e \in U_{1}} N_{e}\left(U_{1}\right)\right) \wedge\left(\bigwedge_{e \in U_{2}} N_{e}\left(U_{2}\right)\right) \\
& =\tau\left(U_{1}\right) \wedge \tau\left(U_{2}\right) .
\end{aligned}
$$

Thus $\tau$ satisfies the condition $\left(\mathrm{OST}_{2}\right)$. Let $\left\{U_{\alpha}\right\}_{\alpha \in \Gamma} \subset 2^{X}$. If $\cup_{\alpha \in \Gamma} U_{\alpha}=$ $\emptyset$. Then it is obvious that

$$
\tau\left(\cup_{\alpha \in \Gamma} U_{\alpha}\right)=1 \geq \bigwedge_{\alpha \in \Gamma} \tau\left(U_{\alpha}\right)
$$

Now suppose $\cup_{\alpha \in P} U_{\alpha} \neq \emptyset$. Then

$$
\begin{aligned}
N_{p}\left(\cup_{\alpha \in \Gamma} U_{\alpha}\right) & \geq N_{p}\left(U_{\alpha_{0}}\right) \quad\left[\mathrm{By}\left(\mathrm{OSN}_{4}\right)\right] \\
& \geq \bigwedge_{e \in U_{\alpha_{0}}} N_{e}\left(U_{\alpha_{0}}\right)=\tau\left(U_{\alpha_{0}}\right) .
\end{aligned}
$$


Thus

$$
\begin{aligned}
\tau\left(\cup_{\alpha \in \Gamma} U_{\alpha}\right) & =\bigwedge_{p \in \cup_{\alpha \in \Gamma}} U_{\alpha} N_{p}\left(\cup_{\alpha \in \Gamma} U_{\alpha}\right) \\
& \geq \bigwedge_{\alpha \in \Gamma} \tau\left(U_{\alpha}\right) .
\end{aligned}
$$

So $\tau$ satisfies the condition ( $\left.\mathrm{OST}_{1}\right)$. Hence $\tau \in \mathrm{OST}(\mathrm{X})$.

Now we show that the mapping $N_{p}: 2^{X} \rightarrow I$ satisfying the conditions $\left(\mathrm{OSN}_{1}\right) \sim\left(\mathrm{OSN}_{5}\right)$ is exactly the ordinary smooth neighborhood system of $p$ w.r.t. $\tau$.

Let a mapping $M_{p}: 2^{X} \rightarrow I$ be the ordinary smooth neighborhood system of $p$ w.r.t. $\tau$. Then, by Theorem 3.5 and the condition $\left(\mathrm{OSN}_{1}\right)$,

$$
M_{p}(U)=0=N_{p}(U) \quad \text { for each } U \in 2^{X} \text { with } \mathrm{p} \notin \mathrm{U} .
$$

For each $U \in 2^{X}$, let $p \in U$. Then

$$
\begin{aligned}
M_{p}(U) & =\bigvee\left\{\tau(V): V \in 2^{X} \quad \text { and } \quad \mathrm{p} \in \mathrm{V} \subset \mathrm{U}\right\} \quad[\text { By Theorem 3.5] } \\
& \left.=\bigvee_{e \in V} N_{e}(V): V \in 2^{X} \quad \text { and } \quad \mathrm{p} \in \mathrm{V} \subset \mathrm{U}\right\} . \quad[\text { By the definition of } \tau] .
\end{aligned}
$$

It is clear that

$$
\bigwedge_{e \in V} N_{e}(V) \leq N_{p}(V) \quad \text { for } \mathrm{p} \in \mathrm{V}, \text { where } \mathrm{V} \in 2^{\mathrm{X}} .
$$

By the condition $\left(\mathrm{OSN}_{4}\right)$

$$
\bigwedge_{e \in V} N_{e}(V) \leq N_{p}(V) \leq N_{p}(U) \text { for } \mathrm{p} \in \mathrm{V} \subset \mathrm{U} \text {, where } \mathrm{U}, \mathrm{V} \in 2^{\mathrm{X}} .
$$

Thus

$$
\begin{aligned}
M_{p}(U) & =\bigvee\left\{\tau(V): V \in 2^{X} \quad \text { and } \quad \mathrm{p} \in \mathrm{V} \subset \mathrm{U}\right\} \\
& =\bigvee\left\{\bigwedge_{e \in V} N_{e}(V): V \in 2^{X} \text { and } \mathrm{p} \in \mathrm{V} \subset \mathrm{U}\right\} \\
& \leq N_{p}(U) \quad \text { for } \mathrm{p} \in \mathrm{U} \in 2^{\mathrm{X}} .
\end{aligned}
$$


On the other hand,

$$
\begin{aligned}
N_{p}(U) \leq & \bigvee\left\{N_{p}(V) \wedge\left(\bigwedge_{e \in V} N_{e}(V)\right): V \in 2^{X} \text { and } \mathrm{V} \subset \mathrm{U}\right\}\left[\mathrm{By}\left(\mathrm{OSN}_{5}\right)\right] \\
= & {\left[\bigvee\left\{N_{p}(V) \wedge\left(\bigwedge_{e \in V} N_{e}(V)\right): V \in 2^{X} \text { and } \mathrm{p} \in \mathrm{V} \subset \mathrm{U}\right\}\right] } \\
& \vee\left[\bigvee\left\{N_{p}(V) \wedge\left(\bigwedge_{e \in V} N_{e}(V)\right): V \in 2^{X} \quad \text { and } \quad \mathrm{p} \notin \mathrm{V} \subset \mathrm{U}\right\}\right] \\
= & \bigvee\left\{N_{p}(V) \wedge\left(\bigwedge_{e \in V} N_{e}(V)\right): V \in 2^{X} \quad \text { and } \quad \mathrm{p} \in \mathrm{V} \subset \mathrm{U}\right\} \\
& {\left[\operatorname{Since} \mathrm{N}_{\mathrm{p}}(\mathrm{V})=0 \text { for } \mathrm{p} \notin \mathrm{V}\right] } \\
\leq & \bigvee\left\{\bigwedge_{e \in V} N_{e}(V): V \in 2^{X} \text { and } \mathrm{p} \in \mathrm{V} \subset \mathrm{U}\right\} \\
= & M_{p}(U) \text { for } \mathrm{p} \in \mathrm{U} \in 2^{\mathrm{X}} .
\end{aligned}
$$

So $N_{p}(U) \leq M_{p}(U)$ for $p \in U \in 2^{X}$.

Hence, by (3.3), (3.4) and (3.5), $M_{p}=N_{p}$. This completes the proof.

\section{References}

[1] K. C. Chattopadhyay, R. N. Hazra and S. K. Samanta, Gradation of openness : fuzzy topology, Fuzzy Sets and Systems 49(1992), 237-242.

[2] M. S. Cheng, G. P. Chae, K. Hur and S. M. Kim, The lattice of ordinary smooth topologies, Honam Math. J. 33(4)(2011), 453-465.

[3] M. Demirci, Neighborhood structures of smooth topological spaces, Fuzzy Sets and Systems 92(1997), 123-128.

[4] P. K. Lim, B. K. Ryou and K. Hur, Ordinary smooth topological spaces, IJFIS. 12(1)(2012), 66-76.

[5] A. A. Ramadan, Smooth topological spaces, Fuzzy Sets and Systems 48(1992), 371-375.

[6] A. P. Sostak, On a fuzzy topological structure, Rend. Circ. Matem. Palermo Ser. II, 11(1985), 89-103.

[7] M. S. Ying, A new approach for fuzzy topology (I), Fuzzy Sets and Systems 39(1991), 303-321.

\section{Jeong Gon Lee}

Division of Mathematics and Informational Statistics, and Nanoscale Science and Technology Institute, Wonkwang University,

Iksan 570-749, Korea.

E-mail: jukolee@wku.ac.kr 
Pyung Ki Lim

Division of Mathematics and Informational Statistics, and Nanoscale Science and Technology Institute, Wonkwang University,

Iksan 570-749, Korea.

E-mail: pklim@wku.ac.kr

Kul Hur

Division of Mathematics and Informational Statistics, and Nanoscale Science and Technology Institute, Wonkwang University,

Iksan 570-749, Korea.

E-mail: kulhur@wonkwang.ac.kr 